\title{
Zu Besuch im privaten „Palmen-Garten“ von Konstantinos Theodoropoulos
}

\author{
HiLKe STEINECKe
}

\begin{abstract}
In this private garden near Athens, Konstantinos TheOdoropoulos cultivates numerous palm species, most of which are rare in Greek gardens. Even in this warm environment, some species require protection against frost, while others appear to be entirely hardy.
\end{abstract}

\section{Zusammenfassung}

Konstantinos Theodoropoulos kultiviert in seinem privaten Garten bei Athen mehrere Dutzend Palmen, die in griechischen Gärten selten zu sehen sind. Auch auf diesem sonnig-warmen, nur selten frostgefährdeten Gelände benötigen einige Arten Winterschutz. Der Garten und einige Palmen der Sammlung werden vorgestellt.

\section{Einführung}

Während des Aufenthaltes in Griechenland anlässlich der Einladung zur 52. Kifissia Flower Show bei Athen bestand die Möglichkeit, einen privaten Garten, der eine ansehnliche Palmensammlung beherbergt, zu besichtigen (vgl. Steinecke 2006). Der etwa $600 \mathrm{~m}^{2}$ große Garten gehört zum Wochenendhaus von Dr. Konstantinos Theodoropoulos, Mitglied der griechischen Palmen-Gesellschaft. Das abschüssige, nach Süden ausgerichtete Gelände mit Blick auf das Mittelmeer befindet sich im Distrikt Koropia/Attika, etwa eine Autostunde vom Zentrum Athens entfernt. Obwohl hier die Küste stark zersiedelt ist, kann man zwischen den Grundstücken noch auf Reste der ursprünglichen Phrygana-Vegetation treffen. Auf den heißen und trockenen Felsen nur wenige Meter oberhalb des Palmen-Gartens wachsen z. B. Kapernstrauch (Capparis ovata), Dornige Bibernelle (Sarcopoterium spinosum), Dornbusch-Wolfsmilch (Euphorbia acanthothamnos), eine kriechende Glockenblume (Campanula drabifolia), Polei-Gamander (Teucrium polium) und der einjährige Stern-Klee (Trifolium stellatum). Dieses sonnig-heiße, allerdings im Sommer auch sehr trockene Gebiet bietet gute Voraussetzungen dafür, sonnen- und trockenheitsliebende Palmen zu kultivieren.

\section{Artenvielfalt im Garten}

Üblicherweise wird in griechischen Parks und Gärten oder an Straßenrändern nur eine kleine Auswahl an Palmen, die im Mittelmeergebiet winterhart sind, ausgepflanzt. Dazu gehören beispielsweise Kanarische Dattelpalme (Phoenix canariensis), Hanfpalme (Trachycarpus fortunei), Zwergpalme (Chamaerops humilis) oder Petticoatpalme (Washingtonia filifera). Da Fröste auf der attischen Halbinsel nur sehr selten auftreten (so z. B. auch im Frühjahr 2008), im äußersten Extremfall in den letzten Jahren an vier aufeinander folgenden Tagen Temperaturen bis $-4^{\circ} \mathrm{C}$ registriert wurden und es im Großraum Athen i. a. nicht mehr als an zwei Tagen schneit, könnten in dieser Region deutlich mehr Palmenarten im Freien kultiviert werden. Viele Palmenarten überstehen die selten auftretenden leichten Fröste ohne Probleme.

Der Rechtsanwalt und Hobby-Botaniker Konstantinos Theodoropoulos probiert deshalb in seinem Garten die Kultur und Winterhärte verschiedenster Palmen aus. Besonders empfindliche Arten aus warmen, tropischen Gebieten erhalten Winterschutz, einige im Kübel wachsende Palmen überwintern in einem kleinen Kalthaus aus Kunststoff.

Theodoropoulos' Sammlung umfasste vor wenigen Jahren noch etwa 150 Palmenarten, das ist immerhin etwa die Hälfte der im Frankfurter Palmengarten kultivierten Palmen-Spezies. In den letzten für griechische Verhältnisse relativ kalten Wintern sind allerdings einige der wärmebedürftigeren Palmen erfroren, so dass die Sammlung im Jahr 2006 nur etwa 80 Arten umfasste. Die meisten seiner Palmen hat TheoDOROPOuLos als Jungpflanzen gekauft, er versucht aber auch selbst, Samen zur Keimung zu 
bringen. So beherbergt sein Garten auch eine Sabal cf. maritima, die aus einem Samen, der aus dem Athener Nationalgarten stammt, herangezogen wurde.

\section{Verschiedene Palmen}

Auf der Terrasse befinden sich vor allem kleinere Kübel mit noch recht jungen, nicht sehr großen Palmen. Jeder freie Platz wird genutzt, selbst unter den Ölbäumen an der unteren Grundstücksgrenze hat so manche Palme einen Platz gefunden. Da die Töpfe schnell austrocknen und besonders die Jungpflanzen einen relativ hohen Wasserbedarf haben, sich außer am Wochenende aber niemand im Garten aufhält, hat Theodoropoulos ein einfaches, aber wirksames Bewässerungssystem eingerichtet. Fast alle Kübel und ausgepflanzte Palmen werden von einem den ganzen Garten durchziehenden System aus dünnen Kunststoff-Wasserleitungen gezogen. Die Wasserzufuhr erfolgt über den Wasseranschluss im Haus und wird über Computer und Zeitschaltuhr geregelt. Sehr gut halten sich in dem Garten verschiedene SabalArten, darunter beispielsweise die in der Karibik heimische, bis 20 m Höhe erreichende Sabal mauritiiformis sowie S. bermudana. Aufgrund ihrer Frosthärte eignen sich diese stattlichen, sonnenliebenden Fächerpalmen auch für die Freilandkultur in Gebieten mit gemäßigtem Klima. Sabal bermudana ist bereits seit 4 Jahren ausgepflanzt. Eine besonders schöne Palme ist Pritchardia hillebrandi aus Hawaii. Bei uns ist sie aufgrund ihrer fast kreisrunden Fächerblätter eine dekorative Zimmerpalme. Im Großraum Athen scheint sie völlig winterhart zu sein. Denn das hier vorhandene Exemplar hat wie die Sabal-Palme bereits den 3. Winter (mit Frost bis

Abb. 1 (oben): Blick auf die Terrasse mit verschiedenen Palmen-Kübeln. Die Pflanzen sind mit einem Bewässerungssystem verbunden.

Abb. 2 (Mitte): Durch spitze, in den Boden gesteckte Hölzchen sollen Katzen von den jungen Palmen fern gehalten werden.

Abb. 3 (unten): Diese Pritchhardia hillebrandi hat bereits drei Winter im Freien überstanden.
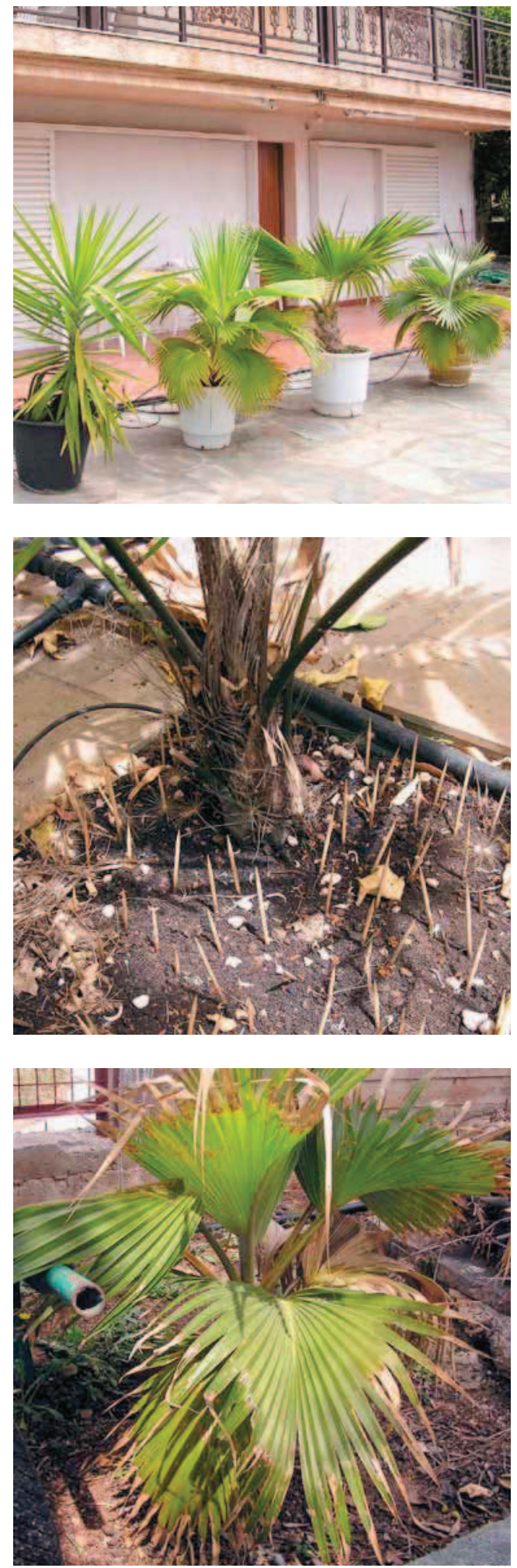


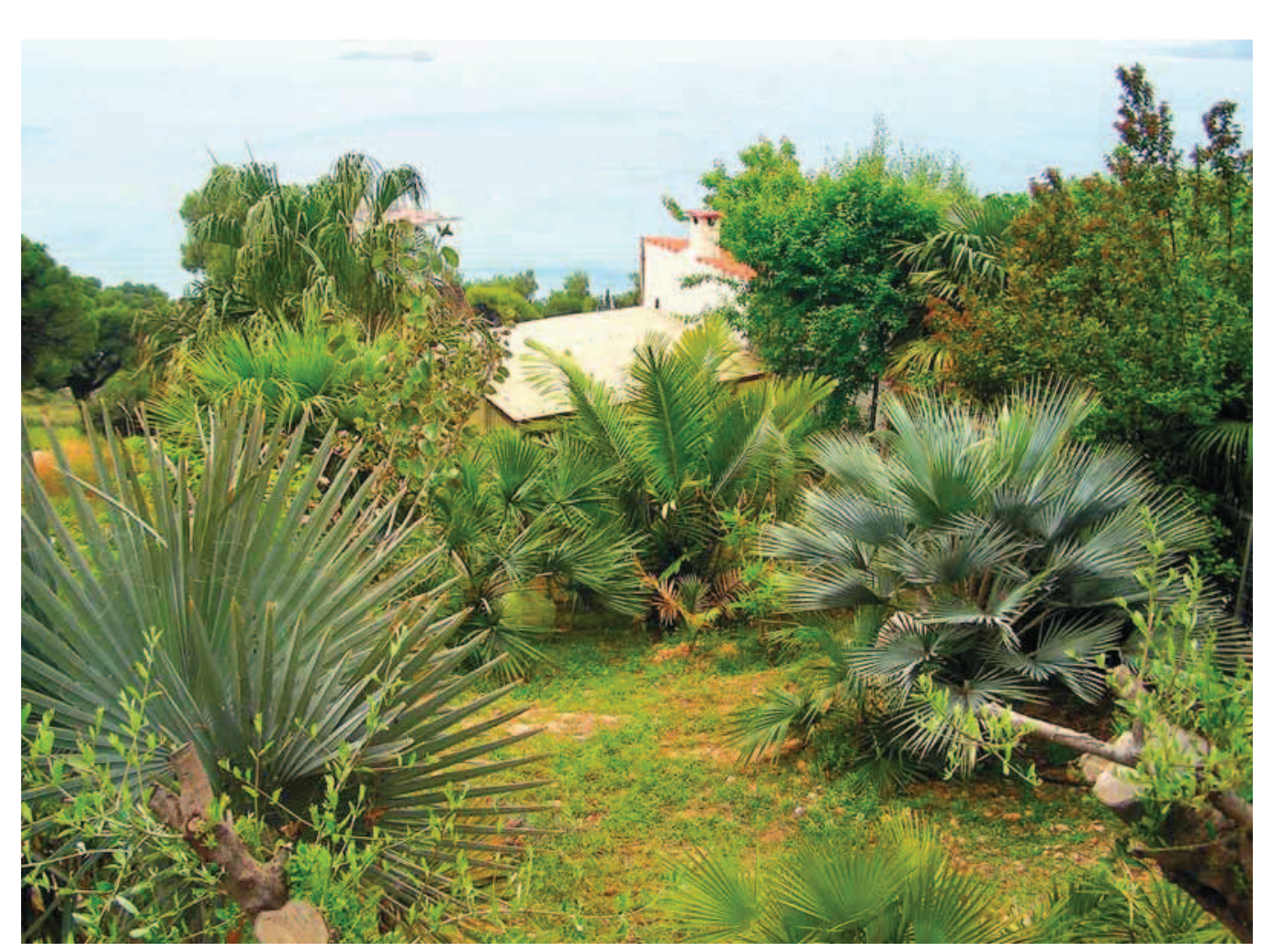

$-4^{\circ} \mathrm{C}$ ) im Freien gut überstanden. $\mathrm{Zu}$ den größeren und bereits etwas älteren Exemplaren im vorderen Gartenbereich gehört eine Chinesische Hanfpalme (Trachycarpus fortunei), eine der wenigen Palmen, die in Europa auch nördlich der Alpen winterhart sind.

TheOdoropoulos sammelt in seinem Garten nicht nur Naturformen, sondern auch interessante Sorten und Varietäten, die sich vor allem in ihrer Form und Färbung der Blätter von den Wildarten unterscheiden. Darunter befinden sich eine Bismarckia nobilis (Heimat: Madagaskar) mit rötlichen Blättern, eine Europäische Zwergpalme (Chamaerops humilis 'Serifera') mit besonders schmalen, blau bereiften Fiedern sowie eine weitere Zwergpalme der Sorte 'Vulcano'.

Seitlich des Hauses ist das Grundstück eher naturbelassen. Zwischen den Gräsern haben sich Wildpflanzen wie Klatschmohn, KronenWucherblume und Windengewächse angesiedelt, die dem Garten im Frühjahr einen hüb- schen bunten Aspekt verleihen. In diesem Teil des Gartens sind die Palmen ausgepflanzt und haben sich zu bereits recht stattlichen Exemplaren entwickelt. Da sich häufig Katzen im Garten aufhalten, die gern unter den jungen Palmen liegen oder daran kratzen und sie somit beschädigen könnten, verwendet THEOdoroPOuLos einen einfachen, aber sehr wirkungsvollen Katzenschutz: Um kleine, durch Katzen gefährdete Palmen hat er dünne Holzstäbe, die an Schaschlikspieße erinnern, wie ein Nadelkissen in den Boden gesteckt.

$\mathrm{Zu}$ den über mannshohen Palmen im Garten gehört eine bereits 9 Jahre im Garten stehende Brahea brandegeei aus Mexiko. Ältere ausgepflanzte Exemplare sollen bis $-7{ }^{\circ} \mathrm{C}$ frosthart sein. Im Gegensatz zu Brahea armata, deren

Abb. 4: Blick über den hinteren Gartenbereich mit den größeren, ausgepflanzten Palmen auf das Mittelmeer. Im Vordergrund blau beblätterte Brahea-Palmen. 
Blätter durch Wachsüberzüge graublau gefärbt sind (eine stattliche $B$. armata ist auch im Halbwüstenhaus des Frankfurter Palmengartens zu besichtigen), bildet $B$. brandegeei grüne Blattfächer. Zusammen mit Livistonia cf. decipiens wurde sie zu einer attraktiven Gruppe gepflanzt, die dem Blick vom Garten auf das Meer einen besonderen Reiz verleiht. Livistonien sind so robust, dass sie gern an der italienischen Adria als Zierbäume gepflanzt werden. Einige weitere Arten aus diesem Gartenbereich sind ein zweites Exemplar einer Bismarckia nobilis, außerdem Brahea armata, Coccothrinax marginata, Bactris setosa, Trithrinax brasiliensis, Jubaea chilensis und Nannorrhops ritchiana. Letztere, auch Mazaripalme genannt, ist in Vorderasien, Indien und Arabien zu Hause. Da sie bis in Höhen um $1600 \mathrm{~m}$ vorkommt und dort im Winter einschneit, dürfte sie für die Freilandkultur im Mittelmeergebiet gut geeignet sein. Eine Kalk liebende Dreizacknadel-Palme (Thrinax campestris) hat in der Mitte des Gartens einen Platz gefunden und ist durch ihre kriechenden, verzweigten Triebe gekennzeichnet. Es versteht sich von selbst, dass die auf Kreta heimische Phoenix theophrastii in einer griechischen Palmensammlung nicht fehlen darf. Obwohl sie angeblich Fröste bis $-9{ }^{\circ} \mathrm{C}$ übersteht und relativ robust sein dürfte, wird sie in Mitteleuropa eher selten kultiviert.

Einige Palmen sind so wüchsig, dass sie bereits blühen und fruchten wie z. B. Sabal etoria und die Geleepalme (Butia capitata). Das Vitamin-C-haltige orangene Fruchtfleisch der aus Südamerika stammenden Geleepalme ist essbar. Anhand zweier nebeneinander gepflanzter Dattelpalmen (Phoenix reclinata, Wild- sowie Kulturform) ist gut zu erkennen, dass durch entsprechende Selektion Kulturpflanzen meist wüchsiger sind als ihre wilden Verwandten. In einer schattigen Ecke hinter dem Haus blüht und fruchtet Chamaedorea radicalis. Bergpalmen vertragen direkte Sonneneinstrahlung eher

Abb. 5 (oben): Konstantinos Theodoropoulos neben einer vor 8 Jahren gepflanzten Brahea brandegeei.

Abb. 6 (unten): Kulturform einer Phoenix reclinata.
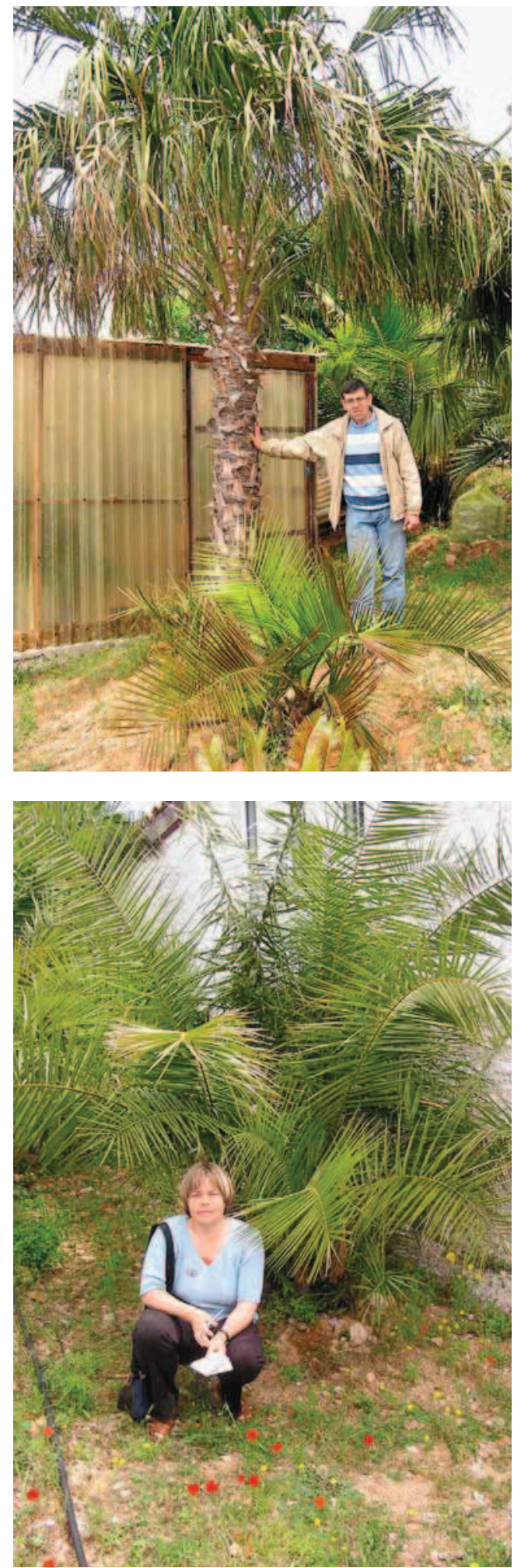

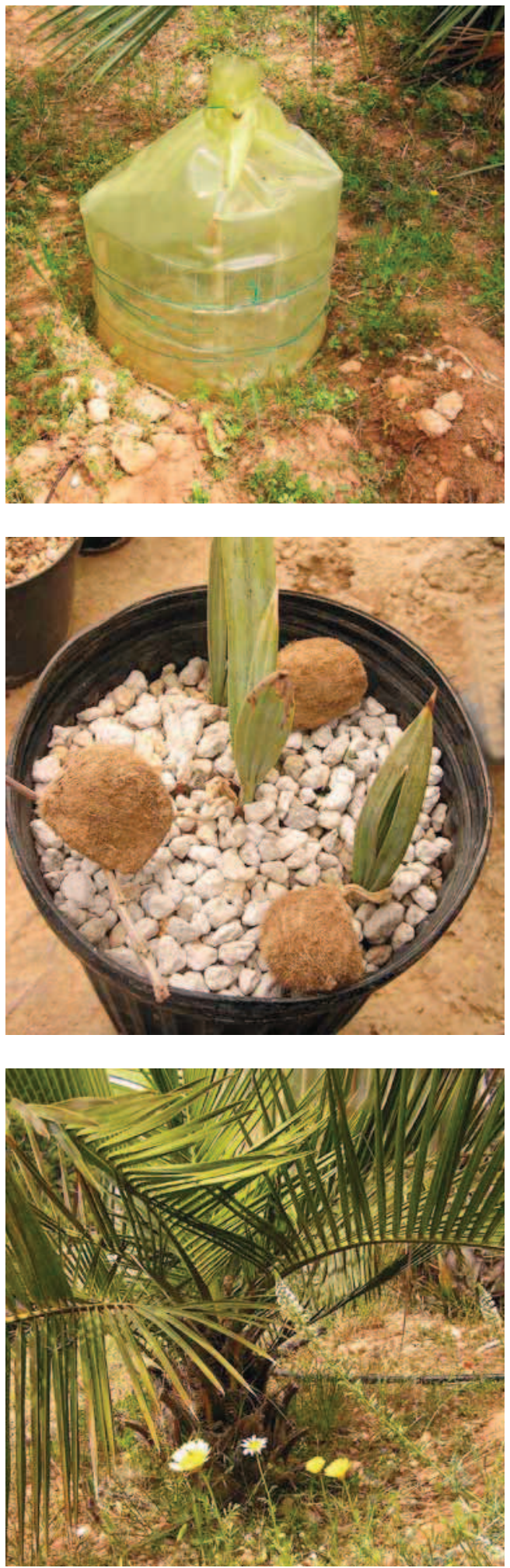

schlecht. Dies verdeutlicht auch ein kleines Exemplar einer Chamaedorea costaricana. Ein in der Nähe dieser Palme stehender schattenwerfender Baum wurde kürzlich ausgelichtet, durch die nun intensivere Sonneneinstrahlung an diesem Standort traten auf den Palmblättern Verbrennungsschäden auf.

\section{Schutz für empfindliche Palmen}

Nicht alle Arten gedeihen unkompliziert. Einerseits nämlich benötigen gerade die Jungpflanzen Wasser, was hier im Sommer Mangelware ist, anderseits könnte Staunässe während einer feuchten Witterungsphase im Winterhalbjahr die Wurzeln schädigen. Deshalb wurde um einige kleine Palmen eine mit Steinen umfasste Vertiefung mit einer kleinen Wasserabflussrinne angelegt.

Für manche Palmen ist die winterliche feucht-kühle Witterung mit seltenen Frösten für ein gutes Wachstum ungeeignet. An den Blättern der Ravenea rivularis beispielsweise sind im Frühling Winterschäden (braune Blätter) zu erkennen. Auch ein Befall durch Spinnmilben weist darauf hin, dass diese Palme im Winter einen Teil ihrer Vitalität eingebüßt hat. Eine ausgepflanzte, kleine und noch empfindlichere Bactris setosa wird deshalb von November bis Ende April durch eine Kunststoff-Haube geschützt. Die empfindlichsten Arten überwintern im Gewächshaus und werden erst, ähnlich wie bei uns, im Mai nach draußen gestellt. Das Kalthaus wird frostfrei gehalten, für die einfache Klimatisierung sorgen ein Thermostat und eine kleine Solarzelle. Das Gewächshaus beherbergt z. B. Ravenea madagascariensis, Hyphaene thebaica, Rhopalostylis baueri und Phoenix roebelenii. Die meisten Palmen, so auch die madagassische Dreikantpalme (Neodypsis decaryi) oder

Abb. 7 (oben): Bactris setosa, im Winterhalbjahr geschützt durch eine Kunststoffhaube.

Abb. 8 (Mitte): Keimpflanzen von Hyphaene thebaica, kultiviert in einem vulkanischen Substrat von Santorini. Die Keimlinge wachsen besser, wenn die Samen durch kleine Stützen über das Substrat angehoben werden.

Abb. 9 (unten): Junge Honigpalme (Jubaea chilensis). 
Reinhardtia paiewonskiana werden in fester Lehmerde aus Kreta kultiviert.

Besonders wärmeliebende Palmen erhalten ein bimsartiges, feinkörniges Kultursubstrat von der Insel Santorini, das in Griechenland im Baustoffhandel erhältlich ist. Sein Vorteil besteht darin, dass es isoliert und vor Kälte schützt, gleichzeitig die Feuchtigkeit gut hält. Das Granulat ist so nährstoffreich, dass sowohl kleine als auch ältere Palmen darin ohne Beimischung von Erde und ohne weitere Düngergaben gedeihen. Dieses vulkanische Substrat wird besonders auch für schwieriger kultivierbare Arten wie die Dumpalme (Hyphaene thebaica) verwendet: Keimlinge von $H$. thebaica entwickeln ihr erstes Blatt erst dann, wenn ihre Wurzel bereits etwa einen halben Meter lang ist. Dementsprechend benötigen sie einen ziemlich tiefen Topf. Bei der Aussaat wird das Pflanzgefäß in der unteren Hälfte mit dem vulkanischen Substrat gefüllt, in das die Wurzeln schnell hineinwachsen. Darüber gibt Theodoropoulos eine Schicht Sand mit den Samen. Nach der Keimung kann auch diese Sandschicht durch das Bimsgranulat ersetzt werden.

\section{Schlussbetrachtung}

Wenn auch der Garten überwiegend Palmen beherbergt, wurden dort auch ein paar andere interessante Gewächse gepflanzt. Von den palmenähnlichen Cycadeen wachsen im Garten Cycas panzhihuaensis mit auffällig blauen Blattstielen und Encephalartos spec. An der oberen Grundstücksgrenze gedeiht das aus Australien und von den Norfolk-Inseln stammende Gehölz Lagunaria patersonii (Malvaceae). Baubinia purpurea und Guave (Psidium guavum) sind Zier- bzw. Nutzbäume im Garten.

Der Besuch dieses privaten „Palmen-Gartens“ hat in eindrucksvoller Weise gezeigt, dass eigentlich viel mehr Palmen als bisher üblich im Mittelmeerraum als freilandtaugliche Zier-

Abb. 10 (oben): Auch Wildpflanzen wie Crysanthemum coronarium finden einen Platz im Garten.

Abb. 11 (unten): Neben den Palmen beherbergt der Garten auch andere Nutz- und Zierpflanzen wie Seerosen und Feigen.
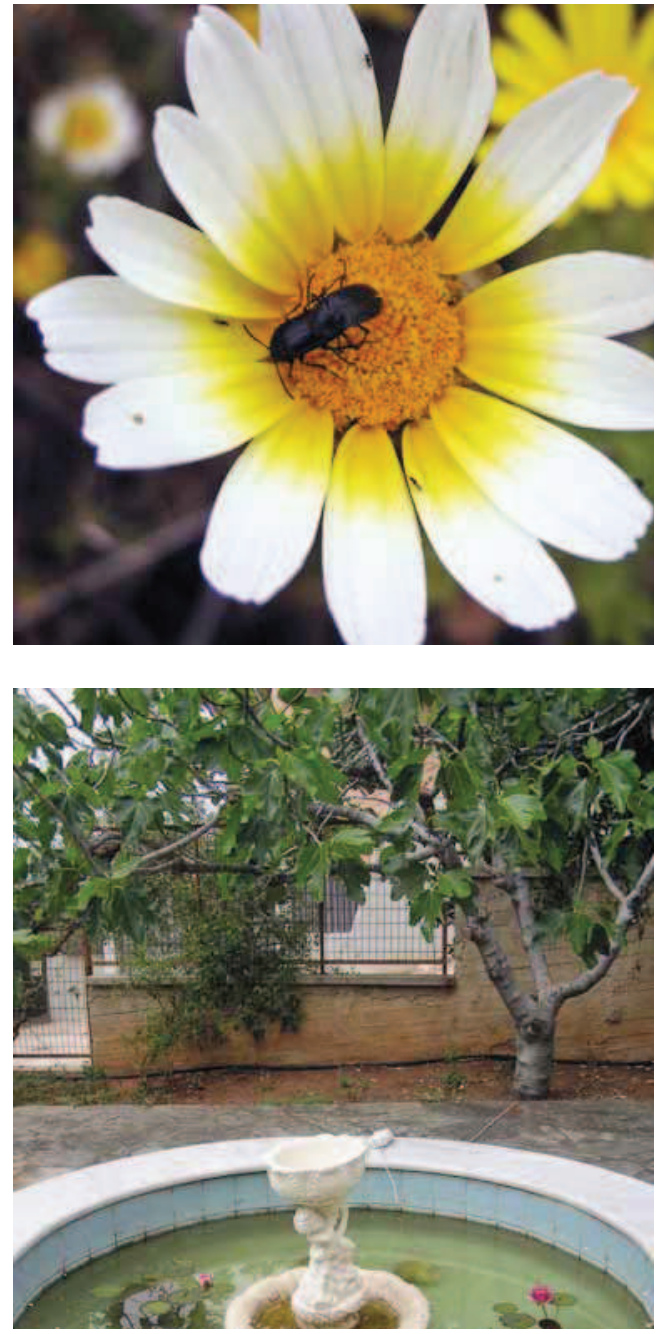

pflanzen verwendet werden könnten. Relativ einfache Konstruktionen können dabei helfen, auch etwas „heikle“ Arten ohne größeren Aufwand besser kultivieren zu können.

Es lohnt sich sicherlich, weiterhin die Winterhärte verschiedenster Palmen zu überprüfen. Dies gilt nicht nur für das klimatisch begünstigte Mittelmeergebiet. Auch im Frankfurter Palmengarten werden Erfahrungen gesammelt, wie verschiedene Palmen, die sich in Mitteleuropa an der Grenze der Winterhärte befinden, mit etwas Winterschutz ganzjährig im Freien kultiviert werden können. 